\section{Decreased cortical spreading depolarizations in neurosurgical patients being given ketamine}

Andrew P Carlson' \& C William Shuttleworth*1

"University of New Mexico School of Medicine, Albuquerque, NM, USA

*Author for correspondence: bshuttleworth@salud.unm.edu

Evaluation of: Hertle DN, Dreier JP, Woitzik J et al.; Cooperative Study of Brain Injury Depolarizations (COSBID). Effect of analgesics and sedatives on the occurrence of spreading depolarizations accompanying acute brain injury. Brain 135(Pt 8), 2390-2398 (2012). This retrospective review by the Cooperative Study of Brain Injury Depolarizations group of 115 patients correlates the occurrence of cortical spreading depolarization (SD), measured with cortical electrodes, with the type of sedation used during monitoring in the intensive care unit. SD has recently been implicated in the progression of acute brain injury, and this study represents the first systematic approach to attempt to understand whether the deleterious effects of SD can be impacted with drug therapy. A significantly decreased probability of SD occurrence was documented with ketamine, but none of the five other drugs that were studied. This effect was seen across all days of monitoring and seemed to have a dose-dependent effect. In evaluating clusters of SD (which most likely contribute to injury progression), ketamine remained significantly associated with decreased likelihood of clusters, along with a weaker effect with propofol and a surprising positive association with midazolam. Owing to the retrospective nature of the study, no causal association can be concluded, although the suppressive effect of NMDA receptor antagonists is consistent with prior animal data. This work will provide critical clinical groundwork for carefully designed clinical trials focused on SD suppression and outcome.

The recognition that cortical spreading depolarizations (SDs) occur in the human brain and are linked to delayed secondary damage after brain injury represents a fundamental paradigm challenge in neurocritical care. The SD process involves a slowly spreading wave of near-complete neuronal and glial depolarization, the mechanisms and consequences of which have been extensively studied in animal models [1]. Particularly important for brain injury is the fact that there is an exceptionally high energy demand following SD, primarily to fuel $\mathrm{Na}^{+} / \mathrm{K}^{+}$-ATPase and other transporters required for restoration of ionic gradients. It is likely that when there are inadequate metabolic substrates available following SD (due to hypoxia, hypoglycemia or oligemia), cells are unable to recover and progress to cell death. SD has been studied in malignant stroke, traumatic brain injury and subarachnoid/ intracerebral hemorrhage, occurring in the majority of monitored patients [1]. Metabolic and clinical data confirm deleterious effects of SD in humans including local brain tissue hypoxia [2], ischemia [3,4] and even worsening clinical outcome [5].

Cooperative Study of Brain Injury Depolarizations (COSBID) is an international consortium that has made substantial progress in the last decade demonstrating the relevance of $S D$ in human brain injury. Participant centers place $1 \times 6$ electrode strips in the presumed penumbral or 'at-risk' cortex at the time of surgery and monitoring for $\mathrm{SD}$ is continued for a number of days in the intensive care unit. From pooled recordings, the COSBID group has made a strong case for the likely involvement of SD in traumatic brain injury, malignant stroke and subarachnoid/intracerebral hemorrhage patients $[1,6]$. The current study by Hertle $e t a l$. represents the first major exploration from the group in terms of therapy for $\mathrm{SD}[7]$.

\section{Methods, results \& analysis}

The study design was a retrospective review of 115 patients with SD monitoring data from participating COSBID centers that also had sedation administration records available. The primary goal was to determine whether the medication choice was associated with any change in SD occurrence. There was a wide amount of heterogeneity in sedative regimens in the group, with 12 different medications reported in the seven centers. To improve statistical power, the authors only included
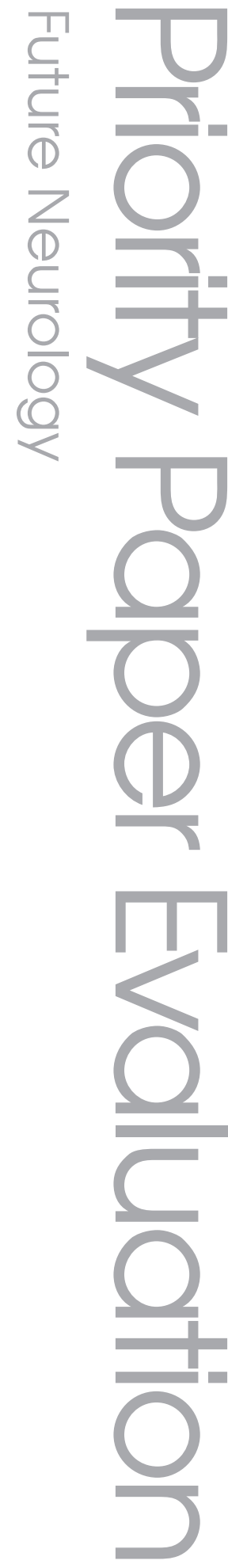

\section{Keywords}

- brain injury = cortical spreading depolarization - cortical spreading depression $=$ neurocritical care $\approx$ sedation

\section{Future : Medicine part of}


the medications with $>1000 \mathrm{~h}$ of combined recording time: midazolam, fentanyl, sufentanyl, ketamine, propofol and morphine.

Since there are many potential confounding factors in a retrospective review, the authors approached the question of effects of medications on SD from a variety of angles. The first approach was to calculate the frequency $(\mathrm{SD} / \mathrm{h})$ for each patient, and then compare the frequencies with and without each of the abovementioned drugs using a simple paired t-test. Only ketamine was found to show a decreased frequency of SD in the 26 patients available. This is demonstrated in Figure 2A of the article by Hertle et al. [7].

One of the most complicated confounders addressed by the authors was the temporal change in SD occurrence and drug administration. While the use of all other medications peaked at approximately 3-5 days after injury, ketamine use peaked at day 7 (shown in Figure 1B of the article by Hertle et al.) [7], suggesting that the use of ketamine may have different indications at participating centers compared with other medications. Nonetheless, the effect of ketamine was evaluated on each day, and was found to be associated with a consistently lower rate of $\mathrm{SD}$, independent of temporal factors (shown in Figure 2B of the article by Hertle $e t a l$.) [7].

The authors recognized that the high degree of variability in the dosing of drugs could alter an observed association with SD frequency, and so constructed a logistic regression model using all the medications. The authors divided the medications administered into quartiles to determine the effect of dosing. Although it is not clear what additional factors might have been included in the regression model or how the quartile dosing was evaluated, ketamine was shown to be the only drug with a significant negative association with SD occurrence (odds ratio [OR]: 0.38; 95\% CI: 0.18-0.79). A negative linear association was also observed between ketamine dose and frequency of $\mathrm{SD} / \mathrm{h}$ (shown in Figure 2C of the article by Hertle et al.) [7].

A different multivariate analysis was then used, focusing on clusters of SDs (which were statistically defined as $>3 \mathrm{SDs}$ in a 3 -h period). Prior studies have reported the deleterious effects of repeated SDs occurring in incompletely recovered tissue $[2,8]$, but no clear definition of an SD cluster had been described until this report. There were 47 clusters reported, and a backward stepwise regression model was used where both ketamine (OR: 0.2; 95\% CI: 0.06-0.64) and propofol (OR: 0.68 , 95\% CI: 0.49-0.95) were found to be associated with decreased odds of SD clusters, while midazolam was surprisingly found to be associated with increased odds of SD clusters (OR: 1.35; 95\% CI: 1.0-1.81). This initial analysis is interesting and supports the general conclusion that ketamine, and perhaps propofol, may prevent these particularly deleterious events; however, future studies will need to analyze clusters on a per patient basis rather than the overall frequency to avoid potential confounding from repeated measures on a single patient.

A variety of preliminary subset data is presented. The authors preface these analyses with a clear acknowledgement that they are underpowered with the data available. The frequency of hours $(n=127)$ of isoelectric depolarization (a strong negative prognostic indicator [9]) was evaluated in 13 patients in a univariate fashion and it was reported that five of the six tested drugs seemed to be associated with decreased SD frequency. In order to test the potential differences in pathophysiology, the effect of medications were assessed for each disease state separately. Although these data were underpowered to draw any firm conclusions, there was a consistent trend that in each case, the probability of SD seems to be less with most drugs compared with no sedation (shown in Figure 3 of the article by Hertle et al.) [7].

Finally, the authors offer us a tantalizingly small piece of clinical outcome data, suggesting that despite these noted effects of ketamine on $\mathrm{SD}$, outcome was worse with higher doses of ketamine. Based on prior work, one might have expected better outcomes when SDs were reduced. While potentially beneficial effects of SD are discussed by the authors, the significant confounding factors in this data set are important to emphasize. For example, it is possible that the ketamine-treated group included those who had developed tolerance to other medications or experienced more severe secondary complications. These types of considerations highlight the importance of future well-controlled prospective intervention studies.

\section{Conclusion \& future perspective}

This study represents a significant advance in hypothesis testing in early human settings regarding therapies directed at SD. The observations that ketamine use is associated with: decreased probability of $\mathrm{SD} / \mathrm{h}$ per patient; that this effect seems to be consistent across the first 15 days after injury; that there 
is a dose-dependent reduction in SD frequency with ketamine; and that ketamine significantly affects the overall odds of occurrence of a cluster of $\mathrm{SD}$, are the most significant findings of the study.

The primary risk of any retrospective review is the temptation to draw conclusions of causality. Even if these data were simple, one could not attribute causality to the drug intervention due to potential confounding factors. This data set is complex and heterogeneous with many potential confounders such as injury severity, reasons for choice of medication and combinations of medications, among others. That said, the authors have examined the effects in a number of different ways to 'build the case' that there may be causality.

This study represents an important step in the field, since mounting evidence is clearly demonstrating the deleterious effects of SD. Multiple types of observations are confirming that SD is not merely a 'harbinger of doom', but is an active participant in the many factors known to cause delayed deterioration or secondary injury after brain injury. If therapies can be developed that target these deleterious effects of SD, a significant impact can likely be made in patient care. In this light, the very preliminary outcome data presented by the authors regarding the negative effect of ketamine dosing on outcome will have to be examined thoroughly before a randomized clinical trial focused on pharmacologic SD suppression with ketamine is considered.

Ketamine's primary effect is as a noncompetitive NMDA receptor (NMDAR) antagonist. The NMDAR has been implicated in propagation of $\mathrm{SD}$, and in animal models NMDAR antagonism [10], as well as ketamine itself [11], have been shown to limit SD and injury expansion. The availability and common use of ketamine at many centers in neurosurgical patients make it an enticing target for further research.

Within the field of SD research, observational data have shown a close correlation between experimental results obtained in animal models and results from previous human trials using a variety of drugs for different conditions such as migraine and subarachnoid hemorrhage [12-17]. These observations suggest that the physiology underlying SD may translate to human studies with relatively higher fidelity than the many previous failed neuroprotective compounds. The recent development of real-time clinical monitoring of SD also raises the possibility of more specific targeting, by using SD incidence as an inclusion criterion for trials, and perhaps even allowing for guided drug applications during SD clusters. As efforts to document and better record the negative effects of SD mount, trials like this by Hertle et al. [7] will be critical in directing multicenter clinical trials focused on both SD effects and outcomes.

\section{Acknowledgements \\ The authors acknowledge helpful input from A Zolyomi, Department of Anesthesiology, University of New Mexico School of Medicine, during the preparation of this article. \\ Financial \& competing interests disclosure \\ CW Shuttleworth is supported by NIH grant NS 51288. The authors have no other relevant affili- ations or financial involvement with any organization or entity with a financial interest in or financial conflict with the subject matter or materials discussed in the manuscript apart from those disclosed. \\ No writing assistance was utilized in the production of this manuscript.}

\section{Executive summary}

\section{Background}

- Cortical spreading depolarization (SD) has been demonstrated in humans after brain injury and is linked to injury expansion.

- It is unknown whether medications given to patients in the neurointensive care unit can inhibit the occurrence of SD.

\section{Methods, results \& analysis}

- Medication administration records were examined for 115 patients who underwent cortical electrode monitoring for SD after neurosurgical procedures.

- Five medications were identified that had over $1000 \mathrm{~h}$ of recording time during administration.

- A consistent effect was noted for decreased frequency of SD with administration of the NMDA receptor antagonist ketamine.

- Possible weaker associations were also noted with propofol and midazolam.

\section{Conclusion \& future perspective}

- This study demonstrates that some current sedation regimens may already have significant effects on preventing SD in the neurointensive care unit.

- These observations will need to be confirmed in larger randomized trials, and raise the question as to whether various other medications should also be investigated for SD suppression, in addition to optimizing current sedation regimens. 


\section{References}

1. Lauritzen M, Dreier JP, Fabricius M, Hartings JA, Graf R, Strong AJ. Clinical relevance of cortical spreading depression in neurological disorders: migraine, malignant stroke, subarachnoid and intracranial hemorrhage, and traumatic brain injury. J. Cereb. Blood Flow Metab. 31(1), 17-35 (2011).

2. Bosche B, Graf R, Ernestus RI et al. Recurrent spreading depolarizations after subarachnoid hemorrhage decreases oxygen availability in human cerebral cortex. Ann. Neurol. 67(5), 607-617 (2010).

3. Offenhauser N, Windmuller O, Strong AJ, Fuhr S, Dreier JP. The gamut of blood flow responses coupled to spreading depolarization in rat and human brain: from hyperemia to prolonged ischemia. Acta Neurochir. Suppl. 110(Pt 1), 119-124 (2011).

4. Dreier JP, Major S, Manning A et al. Cortical spreading ischaemia is a novel process involved in ischaemic damage in patients with aneurysmal subarachnoid haemorrhage. Brain 132(Pt 7), 1866-1881 (2009).

5. Hartings JA, Watanabe T, Bullock MR et al. Spreading depolarizations have prolonged direct current shifts and are associated with poor outcome in brain trauma. Brain 134(5), 1529-1540 (2011).

6. Dreier JP. The role of spreading depression, spreading depolarization and spreading ischemia in neurological disease. Nat. Med. 17(4), 439-447 (2011).

7. Hertle DN, Dreier JP, Woitzik J et al. Effect of analgesics and sedatives on the occurrence of spreading depolarizations accompanying acute brain injury. Brain 135 (Pt 8), 2390-2398 (2012).

8. Dreier JP, Woitzik J, Fabricius M et al. Delayed ischaemic neurological deficits after subarachnoid haemorrhage are associated with clusters of spreading depolarizations. Brain 129(Pt 12), 3224-3237 (2006).

9. Hartings JA, Bullock MR, Okonkwo DO et al. Spreading depolarisations and outcome after traumatic brain injury: a prospective observational study. Lancet Neurol. 10(12), 1058-1064 (2011).

10. Church AJ, Andrew RD. Spreading depression expands traumatic injury in neocortical brain slices. J. Neurotrauma 22(2), 277-290 (2005).

11. Hernandezcaceres J, Maciasgonzalez R, Brozek G, Bures J. Systemic ketamine blocks cortical spreading depression but does not delay the onset of terminal anoxic depolarization in rats. Brain Res. 437(2), 360-364 (1987).

12. Hoffmann U, Dilekoz E, Kudo C, Ayata C. Gabapentin suppresses cortical spreading depression susceptibility. J. Cereb. Blood Flow Metab. 30(9), 1588-1592 (2010).

13. Ayata C, Jin H, Kudo C, Dalkara T, Moskowitz MA. Suppression of cortical spreading depression in migraine prophylaxis. Ann. Neurol. 59(4), 652-661 (2006).

14. Eikermann-Haerter K, Ayata C. Cortical spreading depression and migraine. Curr. Neurol. Neurosci. Rep. 10(3), 167-173 (2010).

15. Ayata C. Spreading depression: from serendipity to targeted therapy in migraine prophylaxis. Cephalalgia 29(10), 1095-1114 (2009).

16. Dietz RM, Weiss JH, Shuttleworth CW. $\mathrm{Zn}^{2+}$ influx is critical for some forms of spreading depression in brain slices. J. Neurosci. 28(32), 8014-8024 (2008).

17. Dreier JP, Windmuller O, Petzold G, Lindauer U, Einhaupl KM, Dirnagl U. Ischemia triggered by red blood cell products in the subarachnoid space is inhibited by nimodipine administration or moderate volume expansion/ hemodilution in rats. Neurosurgery 51(6), 1457-1465; discussion 1465-1457 (2002). 